\title{
Improved Clustering Based Routing Protocol for Wireless Sensor Networks
}

\author{
Naziya Naved Shaikh ${ }^{1}$, Dr. M. U. Kharat ${ }^{2}$ \\ ${ }^{1}$ Department of Computer Engineering, G. H. Raisoni College of Engineering and Mgmt., Wagholi, pune \\ ${ }^{2}$ Department of Computer Engineering, Institute of Engineering, METs Bhujbal Knowledge City, Adgaon, Nashik
}

\begin{abstract}
Minimum range of target teams that we tend to initial broadcast will cause optimum size to search out associate analytical model that cluster size and variety of cs mode; hybrid communication proposes a study of the link between. Again, we've got a centralized agglomeration algorithmic program supported analytical results obtained from the model planned. Finally, we have a tendency to gift a distributed implementation of agglomeration technique. During this project, we tend to even have energy consumption and WSN; safety recently secured and economical information transmission technique victimization the performance are targeted on up. The changed technique of victimization cs was tending to drop the amount of transmissions in device networks. Within the literature major work was done over cs technique is predicated on routing trees. Thus this permits United States of America to planned recent clustering based mostly technique that uses hybrid cs for device networks with goal of up the transmission performance and energy potency. Device nodes are organized in teams. Among a cluster, nodes transmit information to head ache (CH) while not using cs. CHs use cs to transmit information to drop. In our project we tend to are used as a planned SET-IBS algorithmic program that is employed for secure nodes information transmission. SET-IBS algorithmic program consists of 2 phases, one is setup part and second is steadystate introduce every spherical. We introduce the protocol data format and describe the key management of the protocol by exploitation the IBS theme, and also the protocol operations later on.
\end{abstract}

Keywords: Wireless sensor networks, compressive sensing,data collection, clustering

\section{Introduction}

The majority of field experiments data communication contributes to energy consumption of sensor nodes. This sensor network data transmission to reduce the amount of has become an important issue. Compressive sensing (CS) of emerging technology to new frontiers of data collection in sensor networks and sensor networks in target opens in localization.[2] CS broadcasting largely method can reduce the amount of data and Load Balancing traffic across the entire network.

We have hybrid sensor networks for CS uses a clustering method is proposed. Sensor nodes are arranged in groups. Transmit within a cluster nodes CS without using cluster headache $(\mathrm{CH})$ for the data. A data collected all $\mathrm{CHs}$ spanning tree using the method data sync CS to transmit to construct. There should be a cluster to determine how big the hybrid system is an important issue [4]. If the cluster size is too large, cluster $\mathrm{CH}$ sensor nodes to broadcast from within the required number to collect data will be much higher. But if the cluster is very small in size, the large number of groups and data gathering tree to sink all $\mathrm{CHs}$ to transmit their data collected will be bigger, the communication to a huge number of CS will lead using the method. In this consideration, we have an analytical model for the first time that the cluster size number of hybrid communication and CS method, to learn the relationship can lead to find the optimal size of cluster target proposed less number of communication. Again, we have a centralized clustering algorithm based on analytical results obtained from the model proposed. Finally, we present a distributed implementation of clustering method. [5]

Addition of security to LEACH-like protocols is challenging because they dynamically, randomly, and periodically reorganized the network's clusters and data links. Hence, providing constantlong-running node-to-node trust relationships and common key distributions are insufficient for LEACH-like protocols (most existing solutions are provided for distributed WSNs, but not for CWSNs). There are few secure data transmission protocols depending on LEACH-like protocols, such as SecLEACH , GS-LEACH , and RLEACH .[6]

Digital signature is one of the most critical security services offered by cryptography in asymmetric key management systems, where the connection between the public key and the recognition of the signer is obtained via a digital certificate. The identity-based digital signature (IBS) scheme, based on the trouble of factoring integers from identity-based cryptography (IBC), is to derive an entity's public key from its identity information like its name or ID number. A short time ago, the plan of IBS has been developed as a key management in WSNs for security [18].

\section{Literature Survey}

R. Szewczyk, A. Mainwaring, J. Polastre, J. Anderson, and D. Culler Habitat and environmental monitoring are a driving application for wireless sensor networks. They present an analysis of data from a second generation sensor networks deployed during the summer and autumn of 2003. During a 4 month deployment, these networks with 150 devices, produced distinct datasets for both systems and biological analysis. This paper concentrate on nodal and network performance, with emphasize on lifetime, dependability, and the static and dynamic aspects of single and multi-hop networks. They compare the results is collected to expectations set during the design phase: They were able to accurately predict lifetime of the single-hop network, but we ignore the impact of multihoping traffic 


\section{International Journal of Science and Research (IJSR) \\ ISSN (Online): 2319-7064 \\ Index Copernicus Value (2013): 6.14 | Impact Factor (2014): 5.611}

overhearing and the nuances of power source selection. During this time, initially packet loss data was commensurate with lab experiments, over the duration of the reliability, deployment of the backend infrastructure and the transit network had an influential impact on overall network performance.

E. Candes and M. Wakin, Conventional approaches to sampling signals or images follow Shannon's theorem: the sampling rate must be at least twice the maximum frequency present in the signal. In the part of data conversion, standard analog-to-digital converter (ADC) technology plants the usual quantized Shannon representation - the signal is uniformly sampled at or above the Nyquist rate. This article study the theory of compact sampling, also known as compressed sensing or CS, a novel sensing or sampling paradigm that goes versus the common wisdom in data acquisition. CS theory declares that one can recover particular signals and images from far fewer samples or measurements than traditional methods use.

R. Baraniuk, Compressive sensing is a new type of sampling theory, which guesses that sparse signals and images can be reconstructed from what was previously believed to be incomplete information. As a primary feature, efficient algorithms such as? 1-minimization can be used for recovery. The theory has many possible applications in signal processing and imaging. This chapter provides a presentation and overview on theory and numerical aspects of compressive sensing.

J. Haupt, W. Bajwa, M. Rabbat, and R. Nowak, This article describes a very different approach to the decentralized compression of networked data. Considering a specific major aspect of this struggle that revolves around large-scale distributed sources of data and their storage, transmission, and retrieval. The job of sending information from one point to another is a common and well-understood phenomenon. But the problem of efficiently transmitting or sharing information from and among a vast number of distributed nodes remains a prominent challenge, mainly because we do not yet have well prepared theories and tools for distributed signal processing, communications, and information theory in large-scale networked systems.

Compressive Data Gathering for Large-Scale Wireless Sensor Networks, This paper presents the first apply compressive sampling theory to sensor data gathering for large scale wireless sensor networks. The prosperous scheme developed in this research is proposed to offer fresh frame of mind for research in compressive sampling applications and large-scale wireless sensor networks. They consider the scenario in which a large number of sensor nodes are densely deployed and sensor readings are spatially correlated. The proposed compressive data collecting is capable to drop down global scale communication cost without specifying intensive calculation or complicated transmission control. The load balancing attribute is capable of expanding the lifetime of the whole sensor network as well as individual sensors. The proposed scheme can cope with abnormal sensor readings gracefully. They also carry out the analysis of the network capacity of the proposed compressive data gathering and validate the analysis through ns-2 simulations.
More significantly, this novel force into less space required data gathering has been tested on real sensor data and the outcomes show the effectivity and robustness of the proposed scheme.

O. Younis, M. Krunz, and S. Ramasubramanian, The largescale deployment of wireless sensor networks (WSNs) and the need for data aggregation necessitate efficient organization of the network topology for the purpose of balancing the load and prolonging the network lifetime. Clustering has proven to be an effective approach for organizing the network into a connected hierarchy. In this article, we highlight the challenges in clustering a WSN, discuss the design rationale of the different clustering approaches, and classify the proposed approaches based on their objectives and design principles. We further discuss several key issues that affect the practical deployment of clustering techniques in sensor network applications.

E. Candes and T. Tao,Suppose we are given a vector $\mathrm{f}$ in a class FsubeRopf ${ }^{\mathrm{N}}$ e.g., a class of digital signals or digital images. How many linear measurements do we need to make about $\mathrm{f}$ to be able to recover $\mathrm{f}$ to within precision epsi in the Euclidean $\left(\mathrm{lscr}_{2}\right)$ metric? This paper shows that if the objects of interest are sparse in a fixed basis or compressible, then it is possible to reconstruct $f$ to within very high accuracy from a small number of random measurements by solving a simple linear program

\section{Proposed Approach Framework and Design}

\section{Architecture}

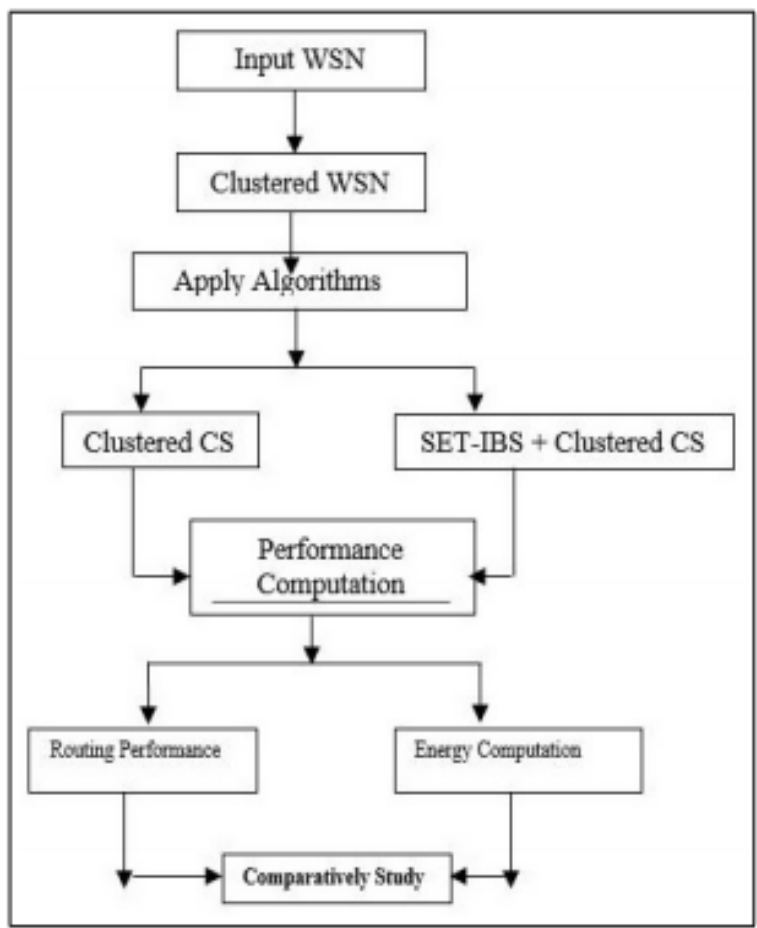

Figure: Proposed System Architecture

\section{Process Flow}

The proposed SET-IBS has a protocol initialization prior to the network deployment and operates in iterations during communication, which consists of a setup stage and a steadystate phase in each iteration. We bring the protocol 


\section{International Journal of Science and Research (IJSR) \\ ISSN (Online): 2319-7064 \\ Index Copernicus Value (2013): 6.14 | Impact Factor (2014): 5.611}

initialization; describe the key management of the protocol by using the IBS scheme, and the protocol operations after that.

\section{Mathematical Model:}

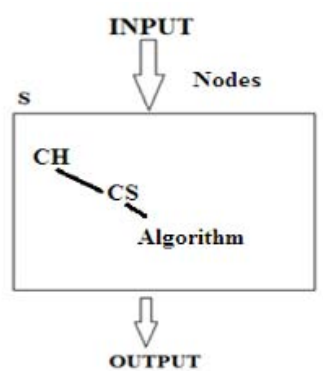

Input $=\{\mathrm{N}, \mathrm{d}\}$

Output $=$ \{reduce data transmission $\}$

Where,

S: System which represented flow of project

$\mathrm{N}$ : nodes

D: data

\section{INPUT:}

Step1: Deploy nodes in wireless sensor network.

Step2: data transmission

$D_{M A X}=\sqrt{\frac{N}{\lambda a^{2}}}$

The value of $\mathrm{D}$ lies in the interval [1, determined later through our analysis.

], and it will be

Step3:- Thus, the number of data transmissions for all sensor nodes within a cluster to transmit their data to the $\mathrm{CH}$ is

$\left(1+\sum_{h=2}^{\frac{D+1}{2}} 8(h-1)\right) \cdot \lambda a^{2}-\left(\frac{D^{3}-D}{3}+D^{2}\right) \cdot \lambda a^{2}$

$\left(\frac{D^{3}-D}{3}+D^{2}\right) \cdot \lambda a^{2} \cdot \frac{N}{\lambda D^{2} a^{2}}=\left(\frac{D}{3}-\frac{1}{3 D}+1\right) \cdot N$

Step4:- Our objective is to minimize total number of transmissions of the hybrid CS method in cluster structure, which is the sum of the intracluster transmissions and the intercluster transmissions.

$$
\begin{aligned}
& T=T_{\text {intra }}+T_{\text {inter }} \\
& =\left(\frac{N}{3}-\frac{M}{2}\right) * D+\left(\frac{N M}{\lambda a^{2}}-\frac{N}{3}\right) * \frac{1}{D} \\
& =\left(\frac{N}{3}-\frac{M}{2}\right) * D+\left(\frac{N M}{\lambda a^{2}}-\frac{N}{3}\right) * \frac{1}{D} \\
& =c_{1} * D+c_{2} * \frac{1}{D}
\end{aligned}
$$

\section{Output:}

Clusters and Energy consuming

\section{Work Done}

In this section we are discussing the practical environment, scenarios, performance metrics used etc.

Input: In this Node and Data is the input for our practical experiment.

Hardware Requirements:

Processor : Pentium IV $2.6 \mathrm{GHz}$

Ram : $512 \mathrm{Mb}$

Hard Disk : 20 GB

Software Requirements:

Front End : J2SE

Back End : MySQL 5.1

Tools Used : Eclipse IDE used

Operating System: Windows 7/8

\section{Results of Practical Work:}

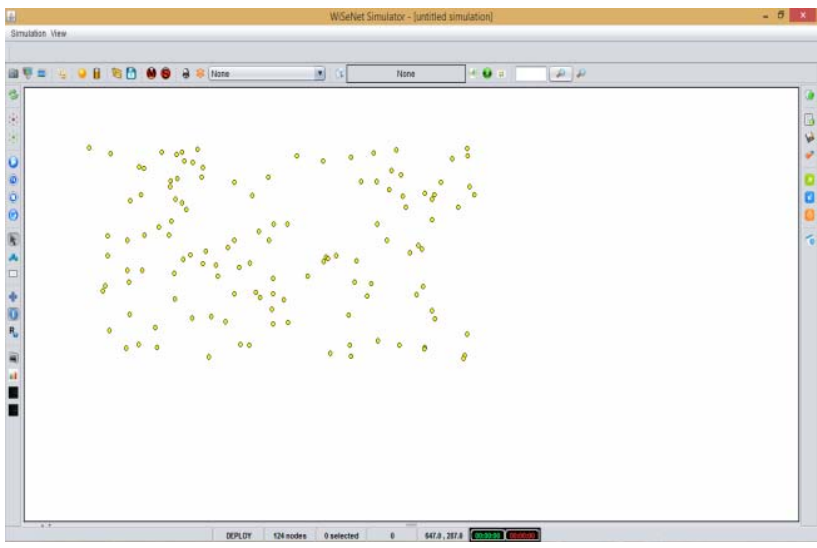

Figure: In this screen we are deploy a number of node

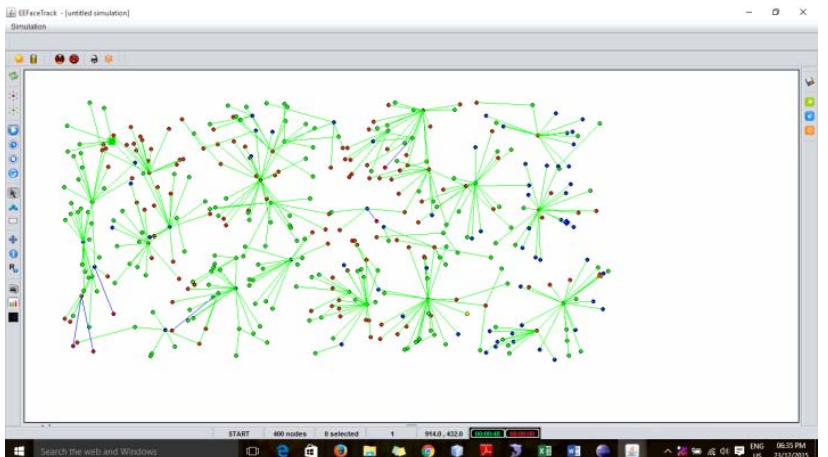

Figure: In this screen a number of nodes are connect and communicate to the sink

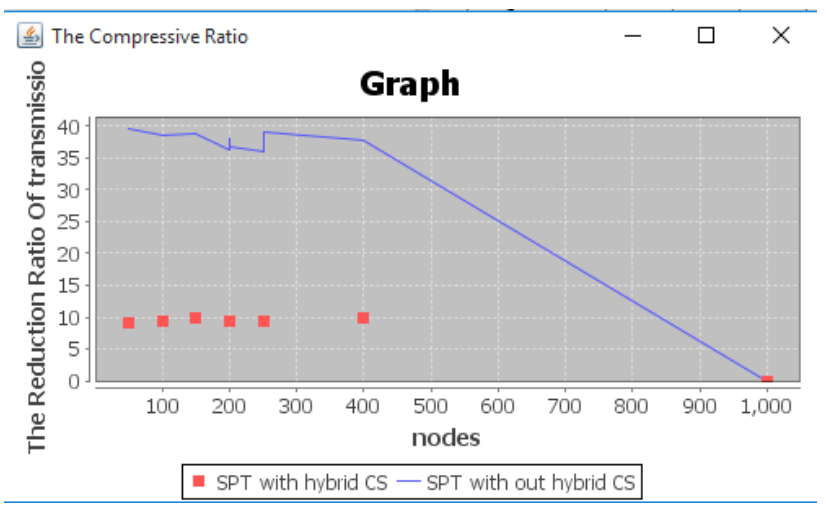

Figure: Compressive Graph 


\section{International Journal of Science and Research (IJSR) \\ ISSN (Online): 2319-7064 \\ Index Copernicus Value (2013): 6.14 | Impact Factor (2014): 5.611}

\section{Conclusion and Future Work}

This research work in addressing the problems of WSNs are going to research in WSNs security and data related to the aggregation. WSNs both problem domain. The major impact of recent efficiency in many ways either to achieve efficient data aggregation or come for effective protection. However, In this project we are presenting new hybrid method which combines two advantages from us recently are going to WSNs security methods and improved security in network data aggregation method as well as for obtaining routing performance of WSNs. In addition of this project we are add the SET-IBS method for secure transmission. In the evaluation section, we provided feasibility of the proposed SET-IBS with respect to the security requirements and analysis against routing attacks.

\section{References}

[1] R. Szewczyk, A. Mainwaring, J. Polastre, J. Anderson, and D. Culler, "An Analysis of a Large Scale Habitat Monitoring Application," Proc. ACM Second Int'l Conf. Embedded Networked Sensor Systems (SenSys '04), pp. 214-226, Nov. 2004.

[2] E. Candes and M. Wakin, "An Introduction to Compressive Sampling," IEEE Signal Processing Magazine, vol. 25, no. 2, pp. 21- 30, Mar. 2008.

[3] R. Baraniuk, "Compressive Sensing [Lecture Notes]," IEEE Signal Processing Magazine, vol. 24, no. 4, pp. 118-121, July 2007.

[4] D. Donoho, "Compressed Sensing," IEEE Trans. Information Theory, vol. 52, no. 4, pp. 1289-1306, Apr. 2006.

[5] J. Haupt, W. Bajwa, M. Rabbat, and R. Nowak, "Compressed Sensing for Networked Data," IEEE Signal Processing Magazine, vol. 25, no. 2, pp. 92-101, Mar. 2008.

[6] C. Luo, F. Wu, J. Sun, and C.W. Chen, "Compressive Data Gathering for Large-Scale Wireless Sensor Networks," Proc. ACM MobiCom, pp. 145-156, Sept. 2009.

[7] S. Lee, S. Pattem, M. Sathiamoorthy, B. Krishnamachari, and A. Ortega, "Spatially-Localized Compressed Sensing and Routing in Multi-Hop Sensor Networks," Proc. Third Int'l Conf. GeoSensor Networks (GSN '09), pp. 11-20, 2009.

[8] C. Luo, F. Wu, J. Sun, and C.W. Chen, "Efficient Measurement Generation and Pervasive Sparsity for Compressive Data Gathering," IEEE Trans. Wireless Comm., vol. 9, no. 12, pp. 728-3738, Dec. 2010.

[9] J. Luo, L. Xiang, and C. Rosenberg, "Does Compressed Sensing Improve the Throughput of Wireless Sensor Networks?" Proc. IEEE Int'l Conf. Comm (ICC), pp. 16, May 2010.

[10] L. Xiang, J. Luo, and A. Vasilakos, "Compressed Data Aggregation for Energy Efficient Wireless Sensor Networks," Proc. IEEE Sensor, Mesh, and Ad Hoc Comm. and Networks (SECON '11), pp. 46- 54, June 2011.

[11]F. Fazel, M. Fazel, and M. Stojanovic, "Random Access Compressed Sensing for Energy-Efficient Underwater Sensor Networks," IEEE J. Selected Areas Comm., vol. 29, no. 8, pp. 1660-1670, Sept. 2011.
[12] J. Wang, S. Tang, B. Yin, and X.-Y. Li, "Data Gathering in Wireless Sensor Networks through Intelligent Compressive Sensing," Proc. IEEE INFOCOM, pp. 603611, Mar. 2012.

[13] B. Zhang, X. Cheng, N. Zhang, Y. Cui, Y. Li, and Q. Liang, "Sparse Target Counting and Localization in Sensor Networks Based on Compressive Sensing," Proc. IEEE INFOCOM, pp. 2255-2263, Apr. 2011.

[14] E. Candes, J. Romberg, and T. Tao, "Robust Uncertainty Principles: Exact Signal Reconstruction from Highly Incomplete Frequency Information," IEEE Trans. Information Theory, vol. 52, no. 2, pp. 489-509, Feb. 2006.

[15]E. Candes and T. Tao, "Near-Optimal Signal Recovery from Random Projections: Universal Encoding Strategies?" IEEE Trans. Information Theory, vol. 52, no. 12, pp. 5406-5425, Dec. 2006.

[16] J. Tropp and A. Gilbert, "Signal Recovery from Random Measurements via Orthogonal Matching Pursuit," IEEE Trans. Information Theory, vol. 53, no. 12, pp. 46554666, Dec. 2007.

[17] M. Youssef, A. Youssef, and M. Younis, "Overlapping Multihop Clustering for Wireless Sensor Networks," IEEE Trans. Parallel and Distributed Systems, vol. 20, no. 12, pp. 1844-1856, Dec. 2009.

[18] S. Soro and W.B. Heinzelman, "Cluster Head Election Techniques for Coverage Preservation in Wireless Sensor Networks," Ad Hoc Networks, vol. 7, no. 5, pp. 955-972, 2009.

[19] O. Younis, M. Krunz, and S. Ramasubramanian, "Node Clustering in Wireless Sensor Networks: Recent Developments and Deployment Challenges," IEEE Network, vol. 20, no. 3, pp. 20-25, May/ June 2006.

[20] W. Heinzelman, A. Chandrakasan, and H. Balakrishnan, "An Application-Specific Protocol Architecture for Wireless Microsensor Networks," IEEE Trans. Wireless Comm., vol. 1, no. 4, pp. 660- 670, Oct. 2002.

[21] O. Younis and S. Fahmy, "HEED: A Hybrid, EnergyEfficient, Distributed Clustering Approach for Ad Hoc Sensor Networks," IEEE Trans. Mobile Computing, vol. 3, no. 4, pp. 366-379, Oct.-Dec. 2004.

[22] S. Bandyopadhyay and E. Coyle, "An Energy Efficient Hierarchical Clustering Algorithm for Wireless Sensor Networks," Proc. IEEE INFOCOM, vol. 3, pp. 17131723, Mar. 2003.

[23] D. Wang, L. Lin, and L. Xu, "A Study of Subdividing Hexagon-Clustered WSN for Power Saving: Analysis and Simulation, "Ad Hoc Networks, vol. 9, no. 7, pp. 1302-1311, Sept. 2011.Digital representation. 